\title{
VXSlate: Combining Head Movement and Mobile Touch for Large Virtual Display Interaction
}

\section{Conference Paper}

\section{Author(s):}

Le, Khanh-Duy; Tran, Tanh Quang; Chlasta, Karol; Krejtz, Krzysztof; Fjeld, Morten; Kunz, Andreas (D)

Publication date:

2021-03-27

Permanent link:

https://doi.org/10.3929/ethz-b-000476748

Rights / license:

In Copyright - Non-Commercial Use Permitted

Originally published in:

https://doi.org/10.1109/VRW52623.2021.00146 


\title{
VXSlate: Combining Head Movement and Mobile Touch for Large Virtual Display Interaction
}

\author{
Khanh-Duy Le* \\ ABB Corporate Research \\ Krzysztof Krejtz \\ SWPS University \\ of Social Sciences and Humanities
}

\author{
Tanh Quang Tran \\ University of Otago
}

\author{
Karol Chlasta \\ Polish-Japanese Academy \\ of Information Technology
}

\author{
Morten Fjeld \\ University of Bergen, \\ Chalmers University of Technology
}

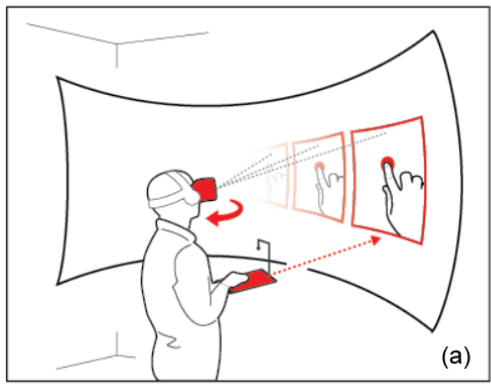

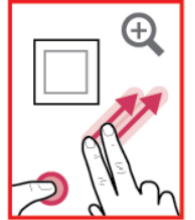

(b)

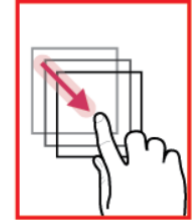

(c)

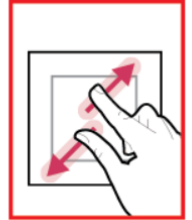

(d)

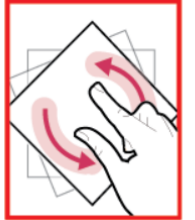

(e)

Figure 1: VXSlate touch interaction: (a) Quickly moving an object as well as the virtual tablet across the large virtual display by touching the object and orienting facing direction towards the target area. (b) Resizing virtual tablet by moving two fingers of one hand while holding the tablet with the other thumb on the screen. (c) Selecting and moving/positioning an object by touching it with a finger. (d) Scaling an object using a two-finger pinch gesture. (e) Rotating an object by a two-finger gesture.

\begin{abstract}
Virtual Reality (VR) headsets can open opportunities for users to accomplish complex tasks on large virtual displays, using compact setups. However, interacting with large virtual displays using existing interaction techniques might cause fatigue, especially for precise manipulations, due to the lack of physical surfaces. We designed VXSlate, an interaction technique that uses a large virtual display, as an expansion of a tablet. VXSlate combines a user's head movements, as tracked by the VR headset, and touch interaction on the tablet. The user's head movements position both a virtual representation of the tablet and of the user's hand on the large virtual display. The user's multi-touch interactions perform finely-tuned content manipulations.
\end{abstract}

Index Terms: Human-centered computing-Human computer interaction-Interaction techniques-Virtual Reality

\section{INTRODUCTION}

Large physical displays, either in the form of a single large screen or multiple tiled screens, have been widely employed to support complex problem-solving tasks. Users can perform interaction techniques on large physical displays, such as selecting objects, moving, scaling and rotating contents using bodily gestures [8], remote pointing [6], or using handheld devices [1] for sketching [3]. However, large physical displays constrain users to stationary and bulky setups. Such setups are quite impractical for home users or for travelers who need to perform these tasks while being out of their office.

Recent progress in Virtual Reality (VR) systems allows users to view and interact with virtual high-resolution contents using a compact hardware setup. This opens new opportunities to further develop virtual environments that include large virtual displays without

*e-mail: duy.le@se.abb.com the need for large physical displays [2]. However, a major weakness of large virtual displays is the lack of a physical surface which allows for intuitive interaction and passive haptic feedback, which can cause user fatigue.

Mobile devices, like tablets, provide multi-touch interactions on smaller surfaces. In addition, such devices can be used for precise distal content manipulations on a small part of a large physical display [5]. Touch tablets can also be used to support precise interactions, such as sketching in Mixed Reality (MR) [10] or rotating and scaling 3D objects in VR [7]. Thanks to passive haptic feedback, tablet screens cause less user fatigue [9]. Interactions on relatively small screens provide more comfort than mid-air gestures [9]. But small screens can result in imprecise interactions on large physical displays when simply up-scaling and mapping interactions. To tackle this problem, head movements are tracked and coupled with the physical tablets, allowing users to manipulate contents on portions of large physical displays [5]. Within current VR systems, head movements (here: right-left and up-down) can easily be registered by applying ray casting from the user's first-person virtual camera. Using off-the-shelf devices, pairing facing direction with multi-touch input can offer users large virtual displays in VR.

In this poster paper, we explore VXSlate ( $\underline{V}$ irtually e $\underline{\boldsymbol{X}}$ tendable $\underline{\text { Slate }})$, a design that considers a large virtual display as an extended surface of a tablet. The design combines head movements, tracked by an off-the-shelf VR system, with input on a touch tablet. This allows a user to perform various manipulations on a large virtual display, ranging from translation, rotation, and scaling of objects to sketching. $V X$ Slate's design was based on previous work on interacting with large physical displays which combine head movements and touch. We then refined it through an iterative process taking into account users' interaction patterns in VR.

\section{Design of VXSLATE}

VXSlate design supports users to perform object manipulations within a range of levels of difficulty on a large virtual display. Using a portable setup, which provides passive haptic feedback, reduces 
users' fatigue. VXSlate combines a user's head movement, tracked by the VR headset, and tablet touch interactions. Interactions using VXSlate consist of two phases: head movements determine the area of interest on the large virtual display, while touches on the tablet are mapped onto the area of interest and directly control contents on the large virtual display (Fig. 1a).

\subsection{Head movement defining the area of interest}

We call the user's area of interest on the large virtual display the virtual tablet. The virtual tablet appears on the large virtual display and is framed as a rectangular area congruent to the tablet. Unintentional head movements can cause instability of the virtual tablet's position, especially in highly precise manipulation tasks like accurate tracing and positioning. Thus, we needed to reduce unintentional movements of the virtual tablet caused by unintended swaying. We chose to detect intentional head movements accordingly. In this approach, we consider angular velocities of head movements in the latest 10 frames. If the average magnitude of angular velocity is greater than around 75 degrees per second and the average change in angular velocity between two consecutive frames is greater than around 0.5 degrees per second (at a 60 frames per second (FPS) in our prototype), head movements are considered to be intentional. When intentional head movements are detected, the virtual tablet repositions. VXSlate also allows users to resize the virtual tablet to suit their task: larger for faster interactions on a large area, smaller for tasks focusing on high precision (Fig. 1b).

\subsection{Touch interaction for direct content manipulation}

Users' touches on the tablet screen are congruently mapped onto the virtual tablet. The touches will directly affect the content on the large virtual display. The user thus can easily apply his/her interactions on commodity mobile devices to work with the large virtual display. To select an object, the user just needs a single touch (Fig. 1c). To scale or rotate an object, the user can perform two-finger pinching or two-finger rotating gestures on the tablet screen (Fig. 1d, 1e). To sketch or trace, the user can select a position of interest on the large virtual display via the tablet and directly perform the task. To move an object, the user can directly select and drag it within the virtual tablet via touch interactions on the tablet (Fig. 1c). VXSlate combines touch and head movements, allowing users to quickly move an object across the large virtual display. In this work, we call this hybrid interaction. The user selects an object with a one-finger touch on the tablet. The selected object then moves with the virtual tablet following the user's head movement to a new area, where the user can fine-tune the position.

While wearing a VR headset, the user cannot see his/her hands on the tablet. Therefore, we chose to represent user hands within VR so that the user can locate his/her hands on the virtual tablet display. Following the MirrorTablet concept [4], we attached a conventional downwards facing camera onto the tablet to capture the user's hands and the background screen. The captured images are processed to remove the background, retaining hands only, which are then mirrored on the virtual tablet (Fig. 1). Since such images do not accurately convey depth information, e.g. when a finger hovers over or touches the screen, the finger's location is highlighted by a red circle on the virtual tablet. To avoid faulty interactions, potentially caused by unintended head movements, the virtual tablet's position will be locked during touch interactions. The virtual tablet's position will be unlocked to follow the user's head movements once there are no touches on the tablet or once ongoing touches cease to move.

\section{EARLY FEEDBACK AND EVALUATION PLAN}

Early qualitative feedback from a pilot study with VXSlate showed that participants felt at ease performing finely-tuned content manipulations, such as rotating or scaling. These manipulations are what user do on their mobile devices. Participants also liked our hybrid interaction; they reported that it helped them quickly move an object across a long distance and then easily fine-tune its position. In a planned user study, we will examine user performance and user perception while using VXSlate. The task will require participants to perform a range of content manipulations at different levels of difficulty, such as selecting, moving, scaling, rotating, and steering. We expect this study to provide further insights into the performance of VXSlate with regards to the variety of manipulation difficulty. Interactions using touch controllers accompanied by the VR headset can be chosen as a baseline to compare performance with VXSlate. To judge performance, we will collect task completion times, task performance accuracy, and time spent on different types of manipulation. We will also examine participants' perceived workload and their perception of the usability of VXSlate as compared to controllers.

\section{ACKNOWLEDGEMENTS}

We thank Barbara Stuckey (editorial), Philippa Beckman (proofreading), Ayça Ünlüer (graphics),Anastasiia Timshina, Kuba Gul (pilot study) and Aditya Giridhar (accompanying video). This research is funded by MediaFutures partners and the Research Council of Norway (grant number 309339).

\section{REFERENCES}

[1] S. Boring, D. Baur, A. Butz, S. Gustafson, and P. Baudisch. Touch projector: mobile interaction through video. In Proceedings of the SIGCHI Conference on Human Factors in Computing Systems, pp. 2287-2296. ACM, New York, NY, USA, 2010.

[2] N. Farahani, R. Post, J. Duboy, I. Ahmed, B. J. Kolowitz, T. Krinchai, S. E. Monaco, J. L. Fine, D. J. Hartman, and L. Pantanowitz. Exploring virtual reality technology and the oculus rift for the examination of digital pathology slides. Journal of pathology informatics, 7:22, 2016.

[3] M. Haller, J. Leitner, T. Seifried, J. R. Wallace, S. D. Scott, C. Richter, P. Brandl, A. Gokcezade, and S. Hunter. The nice discussion room: Integrating paper and digital media to support co-located group meetings. In Proceedings of the SIGCHI Conference on Human Factors in Computing Systems, pp. 609-618. ACM, New York, NY, USA, 2010.

[4] K.-D. Le, K. Zhu, and M. Fjeld. MirrorTablet: exploring a low-cost mobile system for capturing unmediated hand gestures in remote collaboration. In Proceedings of the 16th International Conference on Mobile and Ubiquitous Multimedia, pp. 79-89. ACM, New York, NY, USA, 2017.

[5] M. Nancel, O. Chapuis, E. Pietriga, X.-D. Yang, P. P. Irani, and M. Beaudouin-Lafon. High-precision pointing on large wall displays using small handheld devices. In Proceedings of the SIGCHI Conference on Human Factors in Computing Systems, pp. 831-840. ACM, New York, NY, USA, 2013.

[6] M. Nancel, E. Pietriga, O. Chapuis, and M. Beaudouin-Lafon. Midair pointing on ultra-walls. ACM Transactions on Computer-Human Interaction (TOCHI), 22(5):21, 2015.

[7] H. B. Surale, A. Gupta, M. Hancock, and D. Vogel. Tabletinvr: Exploring the design space for using a multi-touch tablet in virtual reality. In Proceedings of the 2019 CHI Conference on Human Factors in Computing Systems, p. 13. ACM, New York, NY, USA, 2019.

[8] D. Vogel and R. Balakrishnan. Distant freehand pointing and clicking on very large, high resolution displays. In Proceedings of the 18th annual ACM symposium on User interface software and technology, pp. 33-42. ACM, New York, NY, USA, 2005.

[9] S. Vosinakis. Mid-air interaction vs smartphone control for firstperson navigation on large displays: A comparative study. In International Conference on Augmented Reality, Virtual Reality and Computer Graphics, pp. 636-654. Springer, Berlin, Germany, 2018.

[10] M. Xin, E. Sharlin, and M. C. Sousa. Napkin sketch: handheld mixed reality $3 \mathrm{~d}$ sketching. In Proceedings of the 2008 ACM symposium on Virtual reality software and technology, pp. 223-226. ACM, New York, NY, USA, 2008. 